\title{
Antinociceptive effects of intrathecal cimifugin treatment: a preliminary rat study based on formalin test
}

\section{Hyun Young Lee ${ }^{1,2}$, Young Joon $\mathrm{Ki}^{2,3}$, Su Yeong Park', Soo Yeon $\mathrm{Cho}^{2}$, Jinyoung $\mathrm{Seo}^{2}$, Kyung Joon $\mathrm{Lim}^{1,2}$, and Ki Tae Jung ${ }^{1,2}$}

'Department of Anesthesiology and Pain Medicine, School of Medicine, Chosun University, ${ }^{2}$ Department of Anesthesiology and Pain Medicine, Chosun University Hospital, ${ }^{3}$ Department of Medicine, Graduate School, Chosun University, Gwangju, Korea
Received May 5, 2020

Revised June 16, 2020

Accepted August 5, 2020

\author{
Corresponding author \\ Ki Tae Jung, M.D., Ph.D. \\ Department of Anesthesiology and \\ Pain Medicine, Chosun University \\ Hospital, School of Medicine, Chosun \\ University, 365 Pilmun-daero, Dong- \\ gu, Gwangju 61453, Korea \\ Tel: 82-62-220-3223 \\ Fax: 82-62-223-2333 \\ E-mail: mdmole@chosun.ac.kr
}

Background: Cimifugin is one of the components of the root of Saposhnikovia divaricata. The extract derived from $\mathrm{S}$. divaricata is traditionally used as an analgesic. This study was conducted to evaluate the analgesic effect of intrathecal cimifugin in the formalin test.

Methods: Male Sprague-Dawley rats $(n=20)$ were randomized into four groups for intrathecal administration of $70 \%$ dimethylsulfoxide and various doses of cimifugin ( $100 \mu \mathrm{g}, 300$ $\mu \mathrm{g}$, and 1,000 $\mu \mathrm{g})$. The typical flinch response after the injection of $5 \%$ formalin into the hind paw was assessed in two distinct phases: phase 1 until $10 \mathrm{~min}$, and phase 2 from 10 $\min$ to $60 \mathrm{~min} . \mathrm{ED}_{50}$ values were calculated via linear regression.

Results: Intrathecal cimifugin significantly reduced the flinch response in both phases of the formalin test. Significant antinociceptive effects of cimifugin were found with the dose of $300 \mu \mathrm{g}$ in phase 1 and the dose of $100 \mu \mathrm{g}$ in phase 2. The $\mathrm{ED}_{50}$ value (95\% confidence intervals) of intrathecal cimifugin was 696.1 (360.8-1,342.8) $\mu$ g during phase 1 and 1,242.8 (42.0-48,292.5) $\mu$ g during phase 2.

Conclusions: Intrathecal cimifugin has an antinociceptive effect against formalin-induced pain. Cimifugin has an anti-inflammatory effect at low concentrations, and non-inflammatory analgesic effect at higher concentrations.

Keywords: Analgesia; Apiaceae; Chromones; Cimifugin; Nociception; Pain measurement.

\section{INTRODUCTION}

Inflammation is one of the mechanisms of pain. Cytokines and other pro-inflammatory mediators, such as prostaglandins, nitric oxide, serotonin, and histamine, are released from cells after the tissue damage, resulting in pain and hyperalgesia [1]. The inflammatory cascade involves major targets, including cyclooxygenase (COX), interleukins (IL), and tumor necrosis factor- $\alpha$ [2]. Recent interest in chromones as potential candidates for drug development is attributed to their anti-inflammatory effects $[2,3]$.

Saposhnikovia divaricata and Peucedanum japonicum belonging to family Umbelliferae, known as 'Bangpung' in Korea, have been used as traditional herbal medicine to

This is an Open Access article distributed under the terms of the Creative Commons Attribution Non-Commercial License (http://creativecommons.org/licenses/by-nc/4.0) which permits unrestricted non-commercial use, distribution, and reproduction in any medium, provided the original work is properly cited.

Copyright (C) the Korean Society of Anesthesiologists, 2020 
treat fever, rheumatism, neuralgia, and headache [4]. Bioactive substances derived from 'Bangpung' showed antioxidant [5] and anti-inflammatory effects [6,7]. These properties of 'Bangpung' suggested potential pharmaceutical candidates [2]. Compounds isolated from $S$. divaricata, including sec-O-glucosylhamaudol (SOG), hamaudol, ledabouriellol, divaricatol, isofraxidin, and cimifugin showed an analgesic effect [8]. We previously demonstrated the strong antinociceptive effect of intrathecal SOG, which is one of the constituents of $P$. japonicum Thunb. via opioid receptors [9]. Recent studies showed anti-inflammatory effects of cimifugin, a chromone (1,4-benzopyran, Fig. 1), which is one of the bioactive substances derived from $S$. divaricata $[10,11]$. Thus, we hypothesized that the anti-inflammatory effect of cimifugin may result in an analgesic effect. Thus, we conducted a preliminary study to determine the antinociceptive effect of intrathecal cimifugin using a formalin test to confirm the analgesic effect of cimifugin mediated via anti-inflammatory effect. The primary outcome of the study was to the concentration-dependent analgesic effect of cimifugin based on a formalin test. The secondary outcome was calculating the effective dose (ED) of cimifugin required for the analgesia.

\section{MATERIALS AND METHODS}

\section{Animal preparation}

The animal study was approved by the Institutional Animal Care and Use Committee (no. CIACUC 2018-S0042). We followed the guidelines and ethical standards stipulated by the International Association for the Study of Pain [12] and ARRIVE (Animal Research: Reporting of In Vivo Experiments) guidelines (https://www.nc3rs.org.uk/arrive-guidelines) for the investigation of experimental pain in animals. Specific pathogen-free male Sprague-Dawley rats were purchased from Damul Science (Korea), and those weighing 225-250 g each were used in the study $(n=20)$. The rats were housed in

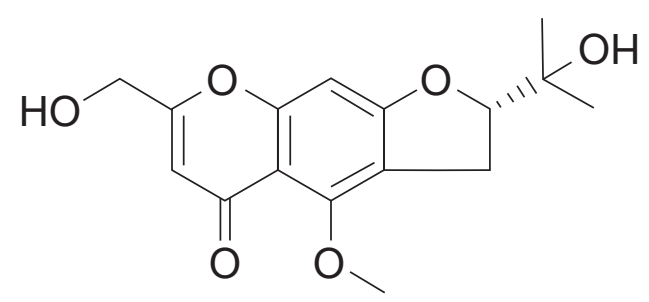

Fig. 1. Chemical structure of cimifugin. the cage located in a room maintained at a constant temperature $\left(20\right.$ to $\left.23^{\circ} \mathrm{C}\right)$, with free access to water and food under a light/dark cycle of 12:12.

\section{Intrathecal catheterization}

The intrathecal catheter was implanted after the anesthesia with isoflurane. After a sterile dressing of the surgical field, the head of the rat at the level of the atlanto-occipital membrane was incised and the cisterna magna was opened. A polyethylene-5 catheter was inserted into the intrathecal space for drug administration [13]. The inserted catheter was placed at the end of the intrathecal catheter at the lumbar enlargement by carefully advancing it into the caudal region about $8.5 \mathrm{~cm}$ from the incision site. The catheter location was confirmed by the leakage of cerebrospinal fluid (CSF) through the tip of the catheter. The catheter tip was pulled out through the skin in the frontal area of the head and fixed with a 3-O silk. The tip of the catheter was plugged with a 30-gauge stainless steel wire to inhibit CSF leakage. After the intrathecal catheterization procedure, the rats were allowed to recover from the anesthesia in the individual cages. Ambulatory function was observed after full recovery from anesthesia. Only the rats without motor or sensory deficits were used in the study. The rats with motor or sensory deficits were euthanized immediately with an overdose of volatile anesthetics.

\section{Drug preparation and grouping}

Cimifugin (purity $\geq 98 \%$ ) was purchased from ChemFaces (China). Cimifugin powder was dissolved in $70 \%$ dimethylsulfoxide (DMSO). The dissolved solutions were diluted to 100, 300, and 1,000 $\mu$ g levels. Cimifugin powder was fully dissolved by DMSO at a concentration greater than $70 \%$. The maximal concentration of cimifugin powder required to dissolve in $70 \%$ DMSO was $1,000 \mu \mathrm{g} / 10 \mu \mathrm{l}$. The concentration of cimifugin was determined by a pilot study (data not shown).

The rats were randomized into four groups (each $\mathrm{n}=5$ ) using a computerized random number according to the study protocol (Fig. 2). The rats in the vehicle-treated group were administered 70\% DMSO intrathecally as a control during the formalin test. The rats in the different test groups were treated with intrathecal cimifugin at concentrations of 100,300 , and $1,000 \mu \mathrm{g}$, respectively, during the formalin test. 


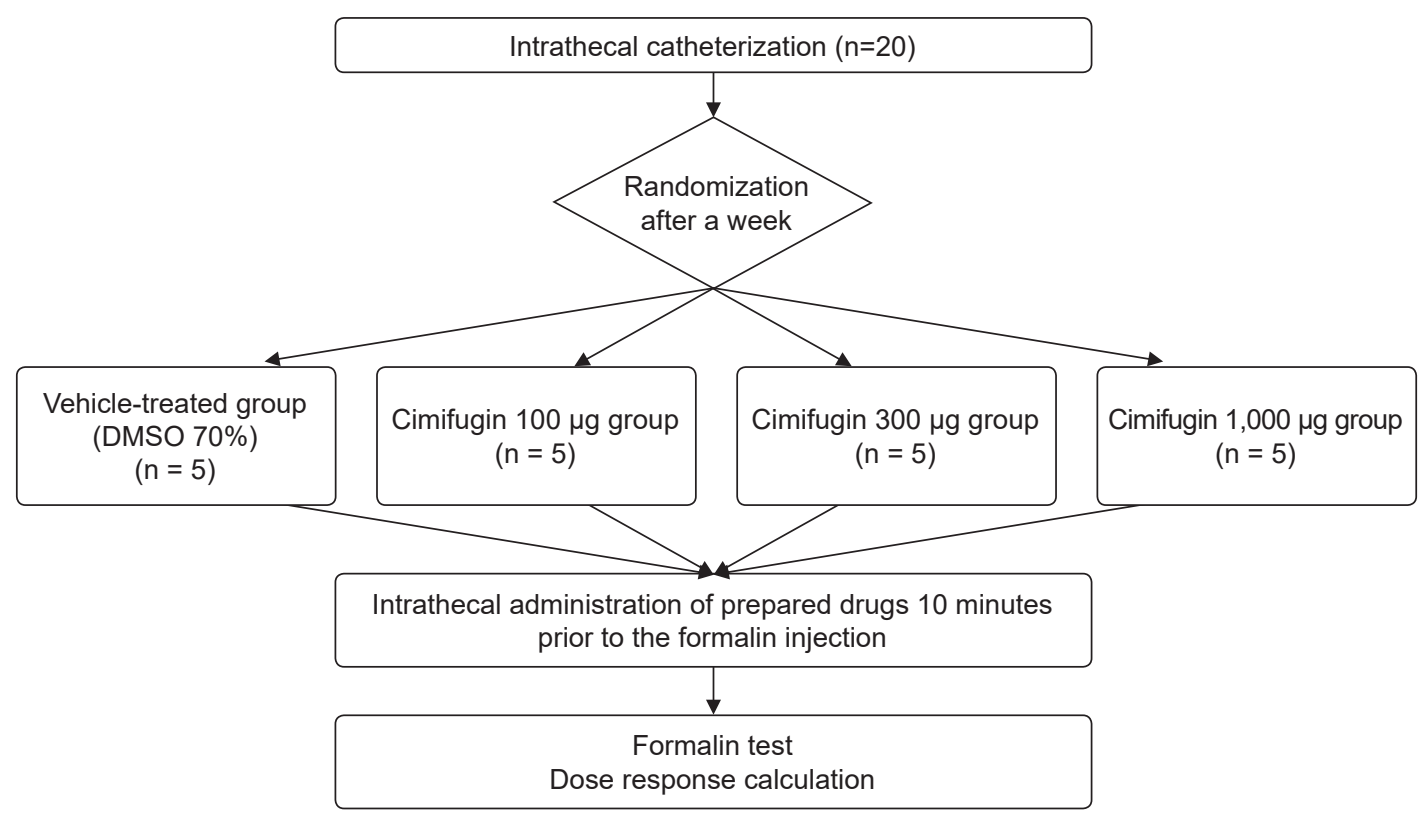

Fig. 2. Flow diagram of the study design. DMSO: dimethylsulfoxide.

\section{Flinch response}

The formalin test was conducted from 9:00 a.m. to 12:00 p.m. by two researchers. One investigator administered formalin injection and intrathecal drug treatment, and another investigator carried out the behavioral test. After adaptation in a restrained cylinder for $20 \mathrm{~min}$, different doses of cimifugin or vehicle (DMSO 70\%) were administered in a volume of $10 \mu \mathrm{l}$ solution intrathecally using a gear-operated Hamilton syringe pump, and $10 \mu \mathrm{l}$ of saline was flushed additionally. After $10 \mathrm{~min}, 5 \%$ formalin $(50 \mu \mathrm{l})$ was subcutaneously injected into the center of the hind paw with a 30 gauge needle. The flinch responses were assessed by counting the number of flinches per minute. Phase 1 response (initial acute phase, 0-9 min) was measured at 1 and $5 \mathrm{~min}$ after the formalin injection, while phase 2 response (10-60 min) was measured every 5 min until an hour after the formalin injection [14].

\section{Statistical analysis}

The sample size was calculated using G*Power 3.1 software. The calculated effect size was 1.12 based on the results of a previous study, which showed significant antinociceptive effect when the total flinch count was decreased to $60.2 \%$ compared with the control [9]. The total sample size in the three groups was calculated as 16 with $\alpha=0.05$ and $95 \%$ power for 13 consecutive flinches based on re- peated measures analysis of variance. We decided the total sample size as 20 given the drop-out rate of $20 \%$.

Dose-responsiveness of cimifugin was calculated as the percentage of control in the two phases as follows.

$\%$ of control $=\left[\frac{\text { sum of phase } 1 \text { or } 2 \text { flinch count with cimifugin }}{\text { sum of control phase } 1 \text { or } 2 \text { flinch count }}\right] \times 100$

The $\mathrm{ED}_{50}$ was defined as a dose of cimifugin that resulted in a $50 \%$ inhibition of flinch count compared with the control. The $\mathrm{ED}_{50}$ was calculated via standard linear regression analysis according to the method of Tallarida [15]. $\mathrm{ED}_{50}$ values and confidence intervals (CIs) in each phase were calculated.

The time-response or the dose-response data of flinch responses are expressed as the number of flinches or the percentage of control. All data are expressed as mean \pm SEM. The flinch responses in phases 1 and 2 were analyzed separately because of the distinct biphasic response of the formalin test. One-way analysis of variance followed by posthoc test with Turkey's test for multiple comparisons was used for the statistical analysis of the flinch responses. $\mathrm{P}$ values less than 0.05 were considered statistically significant.

\section{RESULTS}

Typical biphasic flinch responses to the formalin test were observed in all rats following intrathecal administra- 
tion of vehicle (DMSO 70\%) (Fig. 3).

Pre-treatment with intrathecal cimifugin decreased the flinch response triggered by formalin injection significantly in both phases (Figs. 3, 4). During phase 1, a statistically significant reduction in flinch response was found using the dose of $300 \mu \mathrm{g}$, and the maximal reduction in flinch response was observed at a dose of $1,000 \mu \mathrm{g}$ (41.0\% of control, $\mathrm{P}<0.001$, Figs. 4, 5, and Table 1). During phase 2, a statistically significant reduction of flinch response was found at a dose of $100 \mu \mathrm{g}(65.5 \%$ of control, $\mathrm{P}=0.011)$, and the maximal reduction in flinch response occurred at a dose of $1,000 \mu \mathrm{g}$ (50.6\% of control, $\mathrm{P}=0.003$, Figs. 4,5 , and Table 1).

The $\mathrm{ED}_{50}$ (95\% CIs) values of intrathecal cimifugin were 696.1 (360.8-1,342.8) $\mu \mathrm{g}$ during phase 1 and 1,242.8 (42.0$48,292.5) \mu g$ during phase 2 .

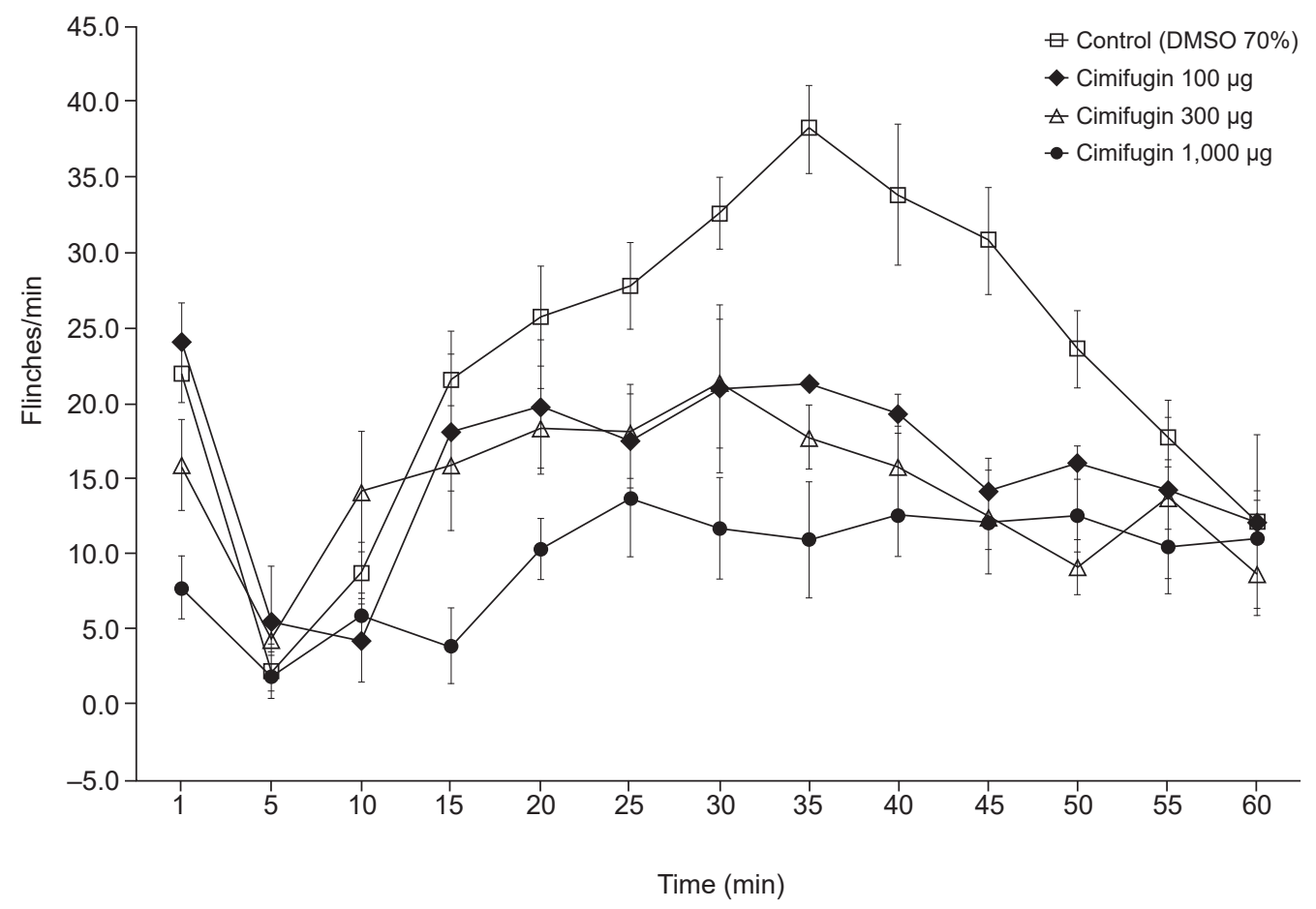

Fig. 3. Time course data showing the antinociceptive effects of cimifugin after formalin injection. Intrathecal cimifugin significantly decreased the formalin-induced flinch response during both phases. DMSO: dimethylsulfoxide. Each line represents the mean \pm SEM of 5 rats/group.
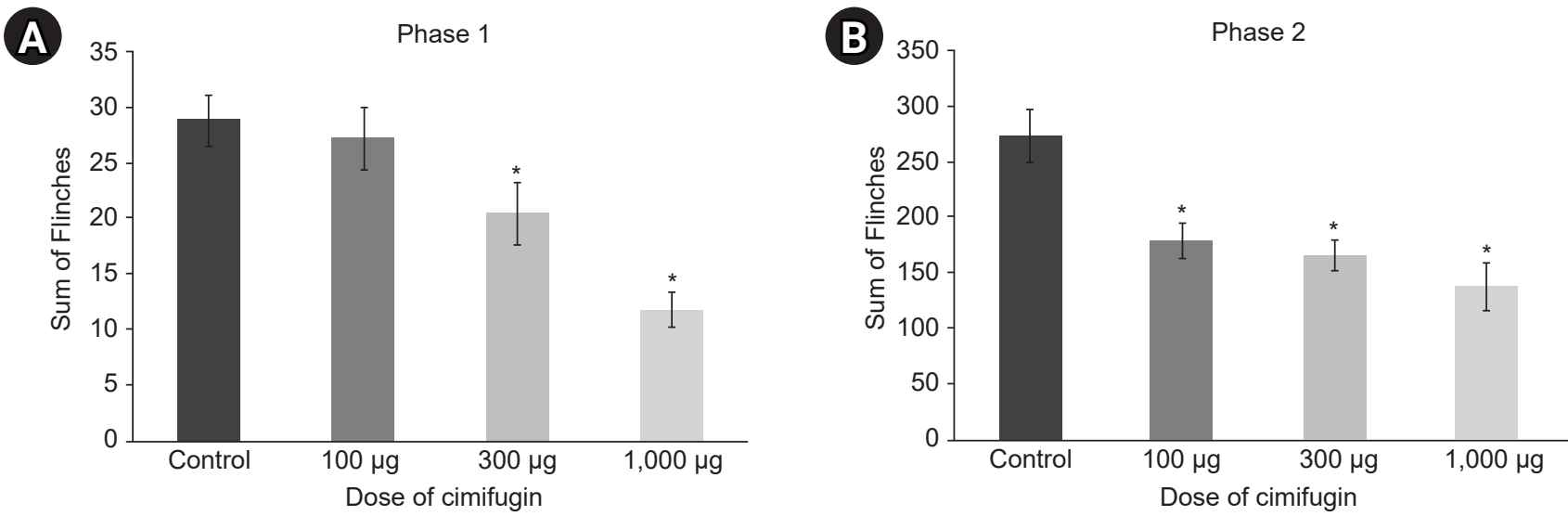

Fig. 4. Antinociceptive effects of intrathecal cimifugin are presented as dose-response data. Intrathecal cimifugin decreased the formalininduced flinch response significantly during phase 1 (A) and phase 2 (B). In phase 1, a statistically significant reduction in flinch response was found with the dose of $300 \mu \mathrm{g}$ and the maximal reduction in flinch response occurred at a dose of 1,000 $\mu \mathrm{g}$. In phase 2 , a statistically significant reduction in flinch response was found at the dose of $100 \mu \mathrm{g}$. The maximal reduction in flinch response occurred at a dose of 1,000 $\mu$ g. Data are expressed as mean \pm SEM. * $P<0.001$ vs. control. 

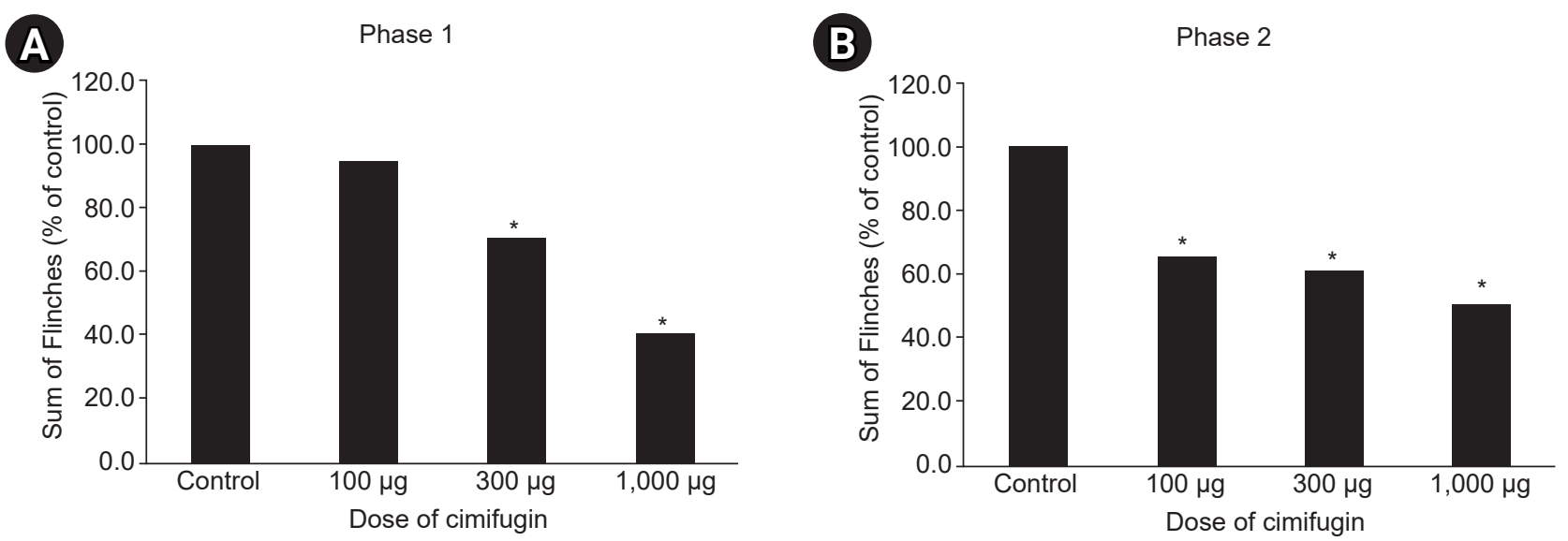

Fig. 5. Dose-response data indicate the antinociceptive effects of intrathecal cimifugin. $* P<0.001$ vs. control.

Table 1. Antinociceptive Effects of Intrathecal Cimifugin: Dose-response Data

\begin{tabular}{|c|c|c|c|c|}
\hline \multirow{3}{*}{ Drugs and dose } & \multicolumn{4}{|c|}{ Dose response (\% of control) } \\
\hline & \multicolumn{2}{|c|}{ Phase 1} & \multicolumn{2}{|c|}{ Phase 2} \\
\hline & $\%$ of control & $P$ value & $\%$ of control & $P$ value \\
\hline Control (DMSO 70\%) & 100.0 & - & 100.0 & - \\
\hline Cimifugin $100 \mu g$ & 94.4 & 0.668 & 65.5 & 0.011 \\
\hline Cimifugin $300 \mu g$ & 70.8 & 0.045 & 60.9 & 0.005 \\
\hline Cimifugin $1,000 \mu g$ & 41.0 & $<0.001$ & 50.6 & 0.003 \\
\hline
\end{tabular}

DMSO: dimethylsulfoxide. P value, compared to the control.

\section{DISCUSSION}

As mentioned above, 'Bangpung' was traditionally used to treat fever and pain. The anti-inflammatory and antioxidant activity of the bioactive substances derived from $S$. $d i$ varicata may correspond to those effects [5-7]. According to the phytochemical studies, Prim-O-glucosylcimifugin is the main chromone present in $S$. divaricata, with antipyretic, anti- inflammatory, and analgesic properties [4]. Cimifugin is an aglycone of prim-O-glucosylcimifugin, synthesized during blood absorption [4]. According to Li et al. [16] cimifugin showed the highest concentration in plasma of rats treated with the extracts of $S$. divaricata orally. They concluded that cimifugin is the potential pharmacodynamic component of $S$. divaricata formed via biotransformation in vivo even though prim-O-glucosylcimifugin is the major constituent $[4,16]$.

As far as we know, only one study reported the analgesic effect of cimifugin. Okuyama et al. [8] reported that oral administration of $80 \mathrm{mg} / \mathrm{kg}$ of cimifugin showed significant analgesia in mice during acetic acid-induced writhing test. The results suggested that the analgesic potency of chromones may be attributed to the non-glycosylated dihydro- pyran-type C-ring. Chromones are an important class of natural products that exhibit antibacterial, anticoagulant, anti-inflammatory, antioxidant, and anticancer activities [3]. Several types of compounds in chromones targeting multiple inflammatory pathways have been reported. The anti-inflammatory effects of chromones are mediated not only via inhibition of the COX pathway but also by neutrophil-dependent superoxide anion generation [3]. A recent study involving RAW264.7 cells showed that cimifugin inhibits the activities of mitogen-activated protein kinase and NF- $\kappa \mathrm{B}$ (nuclear factor kappa-light-chain-enhancer of activated B cells) signaling pathways and suppresses the release of inflammatory factors [11]. Another study of reported that cimifugin significantly attenuated allergic inflammation by reducing thymic stromal lymphopoietin and IL-33 synthesis [10].

These studies suggest that cimifugin has an anti-inflammatory role resulting in an analgesic effect via inhibition of the inflammatory process. Neuroinflammation plays an important role in the generation and modulation of pain [17], so the anti-inflammatory effect of cimifugin was thought to decrease the induction of inflammatory pain. Thus, we conducted the formalin test as a preliminary 
study to evaluate the analgesic effect of intrathecal cimifugin and its relation to inflammation. In the current study, we confirmed that intrathecal administration of cimifugin decreased the flinch response to formalin injection in both phases. The two phases in the formalin test indicate different nociceptive mechanisms [18]. The flinch response in phase 1 is due to a direct effect on the nociceptors, while the phase 2 response represents both inflammatory pain in the peripheral tissue and functional changes in the dorsal horn mediated via inflammatory reaction in the spinal cord. Thus, the inflammatory pathway mediated by prostaglandins does not play an important role during the early phase but the latent phase. However, some nonsteroidal anti-inflammatory drugs such as paracetamol show analgesic effects in both phases, suggesting effects against non-inflammatory pain. In the current study, cimifugin reduced the flinch response in both phases; however, significant antinociceptive effects were observed upon treatment with $300 \mu \mathrm{g}$ in phase 1 (70.8\% of control) and $100 \mu \mathrm{g}$ in phase 2 (65.5\% of control). However, its maximal antinociceptive effect with the $1,000 \mu \mathrm{g}$ dose was more effective in phase 1 (phase 1: $41.0 \%$ of control vs. phase 2: $50.6 \%$ of control).These results are consistent with a previous study reporting the anti-inflammatory effects of isolated compounds from $P$. japonicum Thunb., which showed a relatively weak inhibitory activity of cimifugin against both COX-1 and COX-2 [7]. Therefore, it is suggested that cimifugin has an antinociceptive effect mediated via anti-inflammatory effect at low concentrations and via other non-inflammatory analgesic effects at high concentration.

Other hypotheses have been proposed to explain the mechanisms of action. First, derivatives of natural products display analgesic effect via multiple mechanisms. For example, eugenol, the bioactive compound derived from clove, carries not only anti-inflammatory and antioxidant potential but also analgesic effects mediated via $\alpha_{2}$-adrenergic and opioid receptors [19]. Our previous study investigating SOG, and other extracts derived from $S$. divaricata, showed that SOG exhibits analgesic effect against incisional pain via the $\mu$-opioid receptor [20]. Thus, other targets may modulate acute peripheral nociception. Second, the antioxidant effect may mediate the formalin-induced pain response. According to the study evaluating the analgesic effect of antioxidants in the formalin test, the pro-oxidant may play an important role in the pain induced by tissue injury and intrathecal administration of an antioxidant (4-hydroxy-2,2,6,6-tetramethylpiperidine-1-oxy) effectively reduced the response in both phases [21]. Hong et al. [22] suggested that antioxidants such as vitamin E induce antinociceptive effects by not only decreasing the central sensitization but also scavenging the reactive oxygen species in the peripheral tissue.

Although the current study showed an analgesic effect of cimifugin in both phases of the formalin test, there were several limitations. First, we merely evaluated the pharmacological effect of different doses of cimifugin in the formalin test in this preliminary study, without elucidating the mechanisms of action. Further studies using different routes of administration using antagonists of specific receptors and methods other than formalin test, as well as other molecular studies are needed to determine the exact site of action of cimifugin. Second, a higher dose of cimifugin $(>1,000 \mu \mathrm{g})$ may yield a more potent analgesic effect, but the maximal dose of the current study was 1,000 $\mu \mathrm{g}$. Higher concentrations of cimifugin could not be administered in this study because of technical difficulties and inability to dissolve higher concentrations of cimifugin ( $>1,000 \mu \mathrm{g} / 10 \mu \mathrm{l}$ of $70 \%$ DMSO). Thus, we used 1,000 $\mu \mathrm{g}$ of cimifugin as the maximal dose for the intrathecal administration. Moreover, cimifugin powder was only dissolved in DMSO at a concentration greater than $70 \%$. Even though the control dose of DMSO alone had no analgesic effect, DMSO showedsome reducing effects in the formalin test [23]. The high concentration of DMSO used for drug preparation may affect the study results. Third, the $\mathrm{CI}$ of the $\mathrm{ED}_{50}$ values in phase 2 was large according to the linear regression (42.0 to $48,292.5 \mu \mathrm{g}$ ), which was considered as computational limitation due to the inability to use higher-concentrations of cimifugin. Such a large CI limits further evaluation because of difficulty associated with the intrathecal administration. Further studies investigating the side effects of treatment with large doses are needed. Fourth, further investigations using biological markers are needed for objective results.

In conclusion, intrathecal cimifugin had antinociceptive effects in both phases of the formalin test, suggesting that cimifugin has an anti-inflammatory effect at low concentrations and non-inflammatory analgesic effects at higher concentrations. Further studies are required to investigate the actual mechanisms of cimifugin.

\section{ACKNOWLEDGEMENTS}

This work was supported by the Korean National Research Foundation research grant (NRF-2018R1C1B5 085816). 


\section{CONFLICTS OF INTEREST}

No potential conflict of interest relevant to this article was reported.

\section{AUTHOR CONTRIBUTIONS}

Conceptualization: Ki Tae Jung. Data acquisition: Soo Yeon Cho, Jinyoung Seo, Ki Tae Jung. Formal analysis: Hyun Young Lee, Young Joon Ki, Su Yeong Park. Funding: Ki Tae Jung. Supervision: Ki Tae Jung. Writing-original draft: Hyun Young Lee, Ki Tae Jung. Writing-review \& editing: Kyung Joon Lim, Ki Tae Jung.

\section{ORCID}

Hyun Young Lee, https://orcid.org/0000-0001-5861-3131

Young Joon Ki, https://orcid.org/0000-0001-6203-3690

Su Yeong Park, https://orcid.org/0000-0001-7108-4350

Soo Yeon Cho, https://orcid.org/0000-0002-7828-7908

Jinyoung Seo, https://orcid.org/0000-0002-5256-6132

Kyung Joon Lim, https://orcid.org/0000-0002-3651-0331

Ki Tae Jung, https://orcid.org/0000-0002-2486-9961

\section{REFERENCES}

1. Watkins LR, Milligan ED, Maier SF. Glial activation: a driving force for pathological pain. Trends Neurosci 2001; 24: 450-5.

2. Gautam R, Jachak SM. Recent developments in anti-inflammatory natural products. Med Res Rev 2009; 29: 767-820.

3. Vijaya Bhargavi M, Shashikala P, Sumakanth M. Coumarins and chromones: a remarkable scaffolds for anti-inflammatory activity. J Pharm Sci Res 2017; 9: 1483-9.

4. Li Y, Zhao L, Zhang H, Jia J, Lv L, Zhou G, et al. Comparative pharmacokinetics of prim-O-glucosylcimifugin and cimifugin by liquid chromatography-mass spectrometry after oral administration of Radix Saposhnikoviae extract, cimifugin monomer solution and prim-O-glucosylcimifugin monomer solution to rats. Biomed Chromatogr 2012; 26: 1234-40.

5. Hisamoto M, Kikuzaki H, Ohigashi H, Nakatani N. Antioxidant compounds from the leaves of Peucedanum japonicum thunb. J Agric Food Chem 2003; 51: 5255-61.

6. Zimecki M, Artym J, Cisowski W, Mazol I, Włodarczyk M, Gleńsk M. Immunomodulatory and anti-inflammatory activity of selected osthole derivatives. Z Naturforsch C J Biosci 2009; 64: 361-8.

7. Zheng M, Jin W, Son KH, Chang HW, Kim HP, Bae K, et al. The constituents isolated from peucedanum japonicum Thunb. and their cyclooxygenase (COX) inhibitory activity. Korean J Medicinal Crop Sci 2005; 13: 75-9.

8. Okuyama E, Hasegawa T, Matsushita T, Fujimoto H, Ishibashi M, Yamazaki M. Analgesic components of saposhnikovia root (Saposhnikovia divaricata). Chem Pharm Bull (Tokyo) 2001; 49: $154-60$

9. Kim SH, Jong HS, Yoon MH, Oh SH, Jung KT. Antinociceptive effect of intrathecal sec-O-glucosylhamaudol on the formalin-induced pain in rats. Korean J Pain 2017; 30: 98-103.

10. Wang X, Jiang X, Yu X, Liu H, Tao Y, Jiang G, et al. Cimifugin suppresses allergic inflammation by reducing epithelial derived initiative key factors via regulating tight junctions. J Cell Mol Med 2017; 21: 2926-36.

11. Han B, Dai Y, Wu H, Zhang Y, Wan L, Zhao J, et al. Cimifugin inhibits inflammatory responses of RAW264.7 cells induced by lipopolysaccharide. Med Sci Monit 2019; 25: 409-17.

12. Zimmermann M. Ethical guidelines for investigations of experimental pain in conscious animals. Pain 1983; 16: 109-10.

13. Yaksh TL, Rudy TA. Chronic catheterization of the spinal subarachnoid space. Physiol Behav 1976; 17: 1031-6.

14. Puig S, Sorkin LS. Formalin-evoked activity in identified primary afferent fibers: systemic lidocaine suppresses phase-2 activity. Pain 1996; 64: 345-55.

15. Tallarida RJ, Murray RB. Manual of pharmacologic calculations with computer programs. 2nd ed. New York, Springer. 1987. p. $1-95$.

16. Li Y, Wang H, Chen J, Zhao L, Zhang H, Chai Y, et al. RRLCTOF/MS in identification of constituents and metabolites of Radix Saposhnikoviae in rat plasma and urine. Acad J Second Mil Med Univ 2010; 30: 760-3.

17. Seol TK, Lee W, Park S, Kim KN, Kim TY, Oh YN, et al. Effect of palmitoylethanolamide on inflammatory and neuropathic pain in rats. Korean J Anesthesiol 2017; 70: 561-6.

18. Hunskaar S, Hole K. The formalin test in mice: dissociation between inflammatory and non-inflammatory pain. Pain 1987; 30: 103-14.

19. Park SH, Sim YB, Lee JK, Kim SM, Kang YJ, Jung JS, et al. The analgesic effects and mechanisms of orally administered eugenol. Arch Pharm Res 2011; 34: 501-7.

20. Koh GH, Song H, Kim SH, Yoon MH, Lim KJ, Oh SH, et al. Effect of sec-O-glucosylhamaudol on mechanical allodynia in a rat model of postoperative pain. Korean J Pain 2019; 32: 87-96.

21. Hacimuftuoglu A, Handy CR, Goettl VM, Lin CG, Dane S, Stephens RL Jr. Antioxidants attenuate multiple phases of formalin-induced nociceptive response in mice. Behav Brain Res 2006; 173: 211-6. 
22. Hong BH, Ko YK, Lee YJ, Han K, Kim Y, Lee W. Antinociceptive effects of vitamin $\mathrm{E}$ in formalin-induced nociceptive response in rats. Anesth Pain Med 2011; 6: 59-62.

23. Colucci M, Maione F, Bonito MC, Piscopo A, Di Giannuario A,
Pieretti S. New insights of dimethyl sulphoxide effects (DMSO) on experimental in vivo models of nociception and inflammation. Pharmacol Res 2008; 57: 419-25. 